\title{
VERSO L'UNITÀ DELLE NAZIONI E L'UNITÀ DEI POPOLI
}

\author{
RUMO À UNIDADE DAS NAÇÕES \\ E À UNIDADE DOS POVOS
}

Chiara Lubich

RIASSUNTO: Discorso tenuto da Chiara Lubich, in un Simposio, presso la sede delle Nazioni Unite a New York, il 28 maggio 1997, in cui presenta la sua proposta per la pace e l'unità dei popoli e delle nazioni che oggi è vissuta da milioni di persone, di diverse nazioni, razze, lingue e di diverse religioni e chiese cristiane, anche non credenti, attraverso il Movimento dei Focolari da lei creato in Italia, nel pieno della Seconda Guerra Mondiale. Questo è rappresentato, all'ONU, da uno dei suoi settori che opera nel campo sociale, il New Humanity Movement, che, in quanto organizzazione non governativa, gode di status consultivo di tipo B.

PAROLE CHIAVE: Chiara Lubich; Pace; Movimento dei Focolari; New Humanity Movement

RESUMO: Discurso proferido por Chiara Lubich, em um Simpósio, na Sede das Nações Unidas em Nova York, em 28 de maio de 1997, no qual apresenta sua proposta para a paz e a unidade dos povos e nações que hoje é vivenciada por milhões de pessoas, de diferentes nações, raças, idiomas e de diferentes religiões e igrejas cristãs, inclusive os não-crentes, através do Movimento dos Focolares que ela criou, na Itália, em plena Segunda Guerra mundial. Este se encontra representado, na ONU, por um de seus setores que atua no campo social, o Movimento Humanidade Nova, que por ser uma organização não-governamental, usufrui do status consultivo de tipo.

PALAVRAS-CHAVE: Chiara Lubich; Paz; Movimento dos Focolares; Movimento Humanidade Nova

Eccellenze, Signore e Signori,

Abbiamo l'onore di essere qui nel Centro di un'istituzione mondiale: 1'ONU, che vede simultaneamente presenti come suoi membri 185 Nazioni, "unite" per il fine principale di "mantenere la pace e la sicurezza internazionale".

Una finalità, come recita lo Statuto, percorsa attraverso il rispetto dei diritti fondamentali della persona e dei popoli, la cooperazione economica tra gli Stati, lo sviluppo sociale di ogni Paese. 
Un'azione volta ad eliminare i tanti flagelli che colpiscono l'umanità: guerre, corsa agli armamenti, negazione delle libertà proprie di ogni membro della famiglia umana, e ancora fame, analfabetismo, povertà.

E' questo, lo sappiamo, il nuovo concetto di "pace e sicurezza" che alle Nazioni Unite è stato affidato: la pace cioè intesa non più come assenza di guerra, ma come risultato di condizioni che generano la pace.

Un'istituzione, dunque, grandemente benemerita che trova, oltre il consenso dell'umanità, anche la benedizione del Cielo se è vero quanto ha affermato Gesù Cristo, Principe della Pace: "Beati i costruttori di pace, perché saranno chiamati figli di Dio." (Cf Mt 5,9).

Io, che sono oggi tra loro, rappresento pure una realtà viva praticamente in tutte le nazioni del mondo, anche se più umile, meno nota, ma forse non senza significato nel confronto con la grande conosciutissima istituzione che mi ospita.

Anch'essa infatti, il cui nome è "Movimento dei Focolari", ha per fine la pace nel mondo. E' presente nella Chiesa cattolica e in altre 300 Chiese e in molte Religioni, senza escludere uomini privi forse di un riferimento con l'Eterno, ma di buona volontà.

Puntando sull'unità fra i singoli, fra i gruppi, fra i popoli; sognando una futura realtà, che potrebbe essere espressa dalla parola: Mondo unito, genera la pace nel mondo.

Essa propone, promuove, costruisce la pace non ad alto livello, come è per l'ONU, ma nell'umanità, nel popolo, fra persone diverse per lingua, razza, Nazione, fede.

E quale il vincolo d'unità, causa della pace?

L'amore che batte in fondo ad ogni cuore umano. Che per i seguaci di Cristo può consistere in quella cosiddetta agape che è una partecipazione all' amore stesso che vive in Dio: amore forte, amore capace di amare anche chi non contraccambia ma attacca, come il nemico, amore capace di perdonare.

E per chi segue altre fedi religiose è un amore che può chiamarsi benevolenza ed è espresso da quella "regola d'oro" che impreziosisce molte Religioni e dice: "Fa' agli altri ciò che vorresti fosse fatto a te. Non fare agli altri ciò che non vorresti fosse fatto a te".

Amore che, per le persone che non hanno una fede religiosa, può voler dire filantropia, solidarietà, non-violenza.

Amore, dunque, amore umano-divino che non esclude quello semplicemente umano e lega uomini e donne, bambini e anziani, persone di ogni estrazione sociale, facendole un sol cuore. Questo porta conseguenze sia sul piano spirituale che terreno: una condivisione più o meno piena, ma sempre nell'atmosfera della pace. 
Per questo, per questa sua vocazione all'unità ed alla pace, il Movimento dei Focolari sente oggi, nella mia persona, di trovarsi in questo luogo come nella propria casa e avverte la spinta interiore a offrire la propria collaborazione, che proviene dalla base. Il Focolare, del resto, come molti tra loro sanno, è presente all'ONU attraverso una sua diramazione nel sociale, il Movimento Umanità Nuova, che quale organizzazione non-governativa (ONG) gode dello status consultivo di tipo B presso l'ECOSOC (Consiglio economico sociale).

Questa nostra presenza vuole testimoniare un interesse ed una disponibilità verso tutti coloro che, quali prestigiosi rappresentanti dei popoli all'ONU, o come funzionari altamente qualificati, cercano, spesso con fatica, giorno dopo giorno di conoscere ed interpretare ciò che accade qua e là nel mondo, d'essere presenti dove la pace è ferita, l'equilibrio del mondo compromesso, i diritti umani conculcati, lo sviluppo ridotto ad un lontano obiettivo.

Non solo. Il Movimento dei Focolari oggi nella mia persona avverte anche la spinta a collaborare in altra maniera. Cioè nell'offrire loro una breve panoramica della spiritualità che ci anima ed è il segreto dell'unità e pace fra le persone più varie. Spero che quest'offerta sia loro gradita.

La nostra spiritualità, attuale e moderna, è chiamata spiritualità dell'unità. Essa genera un nuovo stile di vita, assunto da milioni di persone che, ispirandosi fondamentalmente a principi cristiani - senza trascurare, anzi evidenziando, valori paralleli in altre fedi e culture - ha portato in questo mondo, bisognoso di ritrovare o di consolidare la pace, pace e unità.

Questa spiritualità non è vissuta soltanto singolarmente, ma da più persone, insieme. Ha, infatti, una spiccata dimensione comunitaria.

Affonda le sue radici in alcune parole contenute nel Vangelo, che si inanellano l'una nell'altra.

Ne cito qui soltanto alcune.

La spiritualità dell'unità suppone anzitutto una profonda considerazione di Dio per quello che è: Amore, Padre.

Come si potrebbe, infatti, pensare la pace e l'unità nel mondo senza la visione di tutta l'umanità come una sola famiglia? E come vederla tale senza la presenza di un Padre per tutti?

Essa domanda, quindi, di aprire il cuore a Dio Padre, che non abbandona certo i figli al loro destino, ma li vuole accompagnare, custodire, aiutare; che non carica pesi troppo gravosi sulle loro spalle, ma è il primo a portarli.

Credere al suo amore è l'imperativo di questa nuova spiritualità, credere che siamo amati da Lui personalmente e immensamente. 
Egli, infatti, ci conosce nel più intimo, segue ognuno di noi in ogni particolare, conta persino i capelli del nostro capo... (cf $L c$ 12,7). Non lascia alla sola iniziativa degli uomini il rinnovamento della società, ma se ne prende cura.

Credere nell'amore di Dio. E, fra le mille possibilità che l'esistenza offre, scegliere Lui come Ideale della vita. Porsi cioè intelligentemente in quell'atteggiamento verso Dio che ogni uomo assumerà in futuro, quando raggiungerà il destino a cui è stato chiamato: l'Eternità.

Ma, è ovvio, non basta credere all'amore di Dio; non basta aver fatto la grande scelta di Lui come Ideale. La presenza e la premura di un Padre, chiama ognuno ad essere figlio, ad amare a sua volta il Padre, ad attuare giorno dopo giorno quel particolare disegno d'amore che il Padre ha su ciascuno, a fare cioè la Sua volontà.

E si sa che la prima volontà di un padre è che i figli si trattino da fratelli, si vogliano bene, si amino. Conoscano e pratichino quella che può definirsi "l'arte di amare" che troviamo nel Vangelo.

Essa vuole che si ami tutti. Non c'è da scegliere fra simpatico o antipatico, bello o brutto, della mia patria o straniero, bianco o nero o giallo, europeo o americano, africano o asiatico. Utilizzando un linguaggio a loro noto, possiamo dire che l'amore non conosce "alcuna forma di discriminazione". Per un cristiano inoltre tutti vanno amati, perché dietro ad ognuno è Cristo che si ama. Lo dirà Lui stesso: “L'hai fatto a me.” (Cf $M t$ 25,40).

L'arte di amare vuole che si ami per primi, senza aspettare che l'altro ci ami.

Vuole che si ami ognuno come sé. Anche Gandhi diceva: "Tu ed io non siamo che una cosa sola. Non posso farti del male senza ferirmi."

Essa significa ancora saper "farsi uno" con gli altri, cioè far propri i loro pesi, i loro pensieri, le loro sofferenze, le loro gioie.

Per dare un esempio di questo amore rivolto al fratello e della sua efficacia per far cadere barriere erette da secoli e credute incrollabili, vorrei narrare loro una mia esperienza recente.

Mi sono recata quattro mesi fa in Thailandia. Ero stata invitata da monaci buddisti a parlare, a Chiang Mai nel nord del Paese, in un'Università buddista, a studenti e professori e, in un tempio, a monaci, monache e laici buddisti.

Un fatto assolutamente insolito e più sorprendente ancora se pensiamo che mi era stato richiesto di esporre la mia esperienza spirituale notoriamente cristiana.

Come mai ciò?

Lo si può capire se si conoscono i precedenti.

1 WILHELM MÜHS, Parole del cuore, Milano 1996, p. 82 
Un Gran Maestro buddista e un suo discepolo, persona intellettuale ed aperta, in seguito ad aver conosciuto in Asia membri del Movimento, vollero recarsi in Italia in una delle nostre cittadelle, Loppiano, vicino a Firenze, dove i suoi 700 abitanti cercano di vivere con fedeltà il Vangelo che genera pace e unità.

Ne furono profondamente toccati. Toccati dall'amore che hanno trovato.

Il discepolo racconta: "Mettevo le mie scarpe sporche fuori della porta. Al mattino le trovavo pulite.

"Mettevo il mio vestito sporco fuori della porta. Al mattino lo trovavo pulito e stirato.

"Sapevano che avevo freddo perché del sud-est asiatico. Alzavano il

riscaldamento e mi davano coperte...

"Un giorno ho chiesto: 'Perché fate questo?"”

"Perché ti vogliamo bene" fu la risposta che lo sconvolse.

In quella cittadella i due monaci conobbero l'amore cristiano e l'unità, che esso genera fra più persone.

Furono illuminati su diverse verità del cristianesimo e, nell'entusiasmo dell'esperienza fatta, invitarono me a parlare ai loro di questa meraviglia: dell'amore, dell'unità e della pace che ne consegue.

Il mio soggiorno in Thailandia è stato - per grazia di Dio e a Sua gloria - un successo. Una larga strada si è aperta per un dialogo profondo, con arricchimento vicendevole. Anche noi, infatti, abbiamo ammirato la loro buona volontà, la loro vita che ha dell'eroico, la loro saggezza.

Ora si è fatto tutto un piano per proseguire in questo rapporto di fraternità. Ecco, quindi, il valore dell'amore.

$\mathrm{Ma}$, se questo amore è vissuto da più, diventa reciproco.

E Cristo, il "Figlio" per eccellenza del Padre, il Fratello di ogni uomo, halasciato proprio questa norma per l'umanità: l'amore vicendevole. Egli sapeva che era necessario perché ci sia pace e unità nel mondo, perché vi si formi una sola famiglia. Quella famiglia umana universale che supera il limitato concetto di società internazionale, poiché al suo interno i rapporti tra persone, gruppi, popoli, sono pensati per abbattere le divisioni e le barriere, di ogni tipo, in ogni epoca.

Certo, per chiunque si accinga oggi a spostare le montagne dell'odio e della violenza, il compito è immane e pesante. Ma ciò che è impossibile a milioni di uomini isolati e divisi, 
pare diventi possibile a gente che ha fatto dell'amore scambievole, della comprensione reciproca, dell'unità il movente essenziale della propria vita.

E perché questo? C'è un perché.

Un elemento ulteriore di questa nuova spiritualità, conseguente all'amore reciproco, preziosissimo, che sorprende e che meraviglia, è quello annunciato anch'esso dal Vangelo. Dice che, se due o più persone si uniscono nell'amore vero, Cristo stesso, che è la Pace, è presente fra loro e quindi in ciascuno di loro.

E quale garanzia maggiore, quale possibilità superiore può esistere per coloro che vogliono essere strumento di fraternità e di pace?

Questo amore reciproco, questa unità, che tanta gioia dà a chi la mette in pratica, chiede comunque impegno, allenamento quotidiano, sacrificio.

E qui appare, per i cristiani, in tutta la sua luminosità e drammaticità una parola che il mondo non vuole sentire pronunciare, perché ritenuta stoltezza, assurdità, non senso. Questa parola è: croce.

Non si fa nulla di buono, di utile, di fecondo al mondo senza conoscere, senza sapere accettare la fatica, la sofferenza, in una parola senza la croce.

Non è uno scherzo impegnarsi a vivere ed a portare la pace! Occorre coraggio, occorre saper patire.

In fondo non è proprio dal ricordo della sofferenza e del dolore del secondo conflitto mondiale che sorge l'ONU? Dal negativo della divisione e della lotta al positivo dell'integrazione tra gli Stati e dell'unità fra le Nazioni.

E non è un caso che anche il Movimento dei Focolari sia nato in quello stesso momento storico: sotto il buio dei bombardamenti di quella terribile guerra, un gruppo di ragazze scopriva la luce dell'amore reciproco, dell'essere pronte a dare la vita per gli altri e decideva di portare questo amore tra persone, gruppi, popoli, senza escludere o dimenticare nessuno.

Ma, certamente, se più uomini accettassero la sofferenza per amore, lasofferenza che richiede l'amore, essa potrebbe diventare la più potente arma per donare all'umanità la sua più alta dignità: quella di sentirsi non tanto un insieme di popoli l'uno accanto all'altro, spesso in lotta tra loro, ma un solo popolo, abbellito della diversità di ognuno e custode delle differenti identità. 
Dio Padre inoltre col Suo amore ci aiuta sempre in questo arduo cammino.

E vorrei ricordare Maria, la madre di Gesù e di ogni uomo della terra, amata, venerata, presente anche in altre Religioni. A lei si può attingere ispirazione, conforto, sostegno: è compito di una madre comporre e ricomporre sempre la famiglia.

Questa spiritualità comunitaria non è legata necessariamente ad una Chiesa o ad una Religione: è universale e può in qualche modo essere vissuta da tutti.

Per essa, infatti, si sono aperti fecondi dialoghi con tutti gli uomini: con cristiani di molte Chiese, con credenti di diverse Religioni e con persone delle più varie culture, le quali trovano qui sottolineati i valori in cui credono. Insieme ci si avvia a quella pienezza di verità cui tutti tendiamo.

Per essa, per questa spiritualità, oggi uomini e donne di quasi tutte le Nazioni del mondo, lentamente ma decisamente stanno tentando di essere, almeno là dove si trovano, germi di un popolo nuovo, di un mondo di pace, più solidale soprattutto verso i più piccoli, i più poveri, di un mondo più unito.

Per essa sentiamo di dover portare il nostro contributo anche qui, in questa "casa" di incontro fra i popoli, per sostenere con un supplemento d'anima gli sforzi che si vanno compiendo per fare dell'ONU uno strumento adeguato alle attese dell'umanità.

E', del resto, ormai parte del "sentire comune" dei protagonisti della vita internazionale la necessità di rileggere il senso della reciprocità, uno dei cardini dei rapporti internazionali, e che è anche alla base della nostra spiritualità e quindi della nostra azione. Reciprocità, che richiede di superare antiche e nuove logiche di schieramento, stabilendo invece relazioni con tutti come il vero amore esige; che domanda di operare per primo, senza condizioni e attese; che porta a vedere l'altro come un altro se stesso e quindi a pensare in questa linea ogni tipo di iniziativa: disarmo, sviluppo, cooperazione.

Una reciprocità in grado di portare ogni protagonista della vita internazionale a vivere l'altro, i suoi bisogni e le sue capacità, non soltanto nelle emergenze, ma a condividerne quotidianamente l'esistenza.

La pace, come testimoniano anche le finalità e l'azione delle Nazioni Unite, ha nomi nuovi e richiede in primo luogo uno sforzo che l'ONU, con il vostro speciale apporto e il contributo di tutti, può sostenere: superare la categoria del nemico, di qualsiasi nemico.

Escludere la guerra non basta, vanno create le condizioni perché ogni popolo senta di poter amare la patria altrui come la propria, in un reciproco e disinteressato scambio di doni.

Che Dio, Padre di tutti, voglia fecondare questi nostri sforzi, con quelli di quanti sono impegnati all'eccelso fine della pace. E che si possa, come ha detto Giovanni Paolo 
II all'ONU nel cinquantennio della sua fondazione: “... Costruire nel secolo che sta per giungere e per il prossimo millennio una civiltà degna della persona umana, una vera cultura della libertà e della pace.

"Possiamo e dobbiamo farlo! - ha continuato - E facendolo, potremo renderci conto che le lacrime di questo secolo hanno preparato il terreno ad una nuova primavera dello spirito umano." ${ }^{2}$

Grazie, Eccellenze, Signore e Signori, del loro ascolto. Stiano certi che noi non li dimenticheremo, ma li ricorderemo al Principe della pace.

2 "L’Osservatore Romano", 6 ottobre 1995, p. 6-7 